\title{
Novel approaches in cancer treatment: preclinical and clinical development of small non-coding RNA therapeutics
}

Rossana Cuciniello ${ }^{1,2+}$, Stefania Filosa ${ }^{1,2+}$ and Stefania Crispi ${ }^{1 *}$

\begin{abstract}
Short or small interfering RNAs (siRNAs) and microRNA (miRNAs) are molecules similar in size and function able to inhibit gene expression based on their complementarity with mRNA sequences, inducing the degradation of the transcript or the inhibition of their translation.

siRNAs bind specifically to a single gene location by sequence complementarity and regulate gene expression by specifically targeting transcription units via posttranscriptional gene silencing. miRNAs can regulate the expression of different gene targets through their imperfect base pairing.

This process - known as RNA interference (RNAi) - modulates transcription in order to maintain a correct physiological environment, playing a role in almost the totality of the cellular pathways.

siRNAs have been evolutionary evolved for the protection of genome integrity in response to exogenous and invasive nucleic acids such as transgenes or transposons. Artificial siRNAs are widely used in molecular biology for transient silencing of genes of interest. This strategy allows to inhibit the expression of any target protein of known sequence and is currently used for the treatment of different human diseases including cancer.

Modifications and rearrangements in gene regions encoding for miRNAs have been found in cancer cells, and specific miRNA expression profiles characterize the developmental lineage and the differentiation state of the tumor. miRNAs with different expression patterns in tumors have been reported as oncogenes (oncomirs) or tumor-suppressors (antioncomirs). RNA modulation has become important in cancer research not only for development of early and easy diagnosis tools but also as a promising novel therapeutic approach.

Despite the emerging discoveries supporting the role of miRNAs in carcinogenesis and their and siRNAs possible use in therapy, a series of concerns regarding their development, delivery and side effects have arisen.

In this review we report the biology of miRNAs and siRNAs in relation to cancer summarizing the recent methods described to use them as novel therapeutic drugs and methods to specifically deliver them to cancer cells and overcome the limitations in the use of these molecules.
\end{abstract}

Keywords: Cancer therapy, RNA interference, microRNA (miRNA), Small interfering RNA (siRNA), Nanoparticles, sncRNAs therapeutics

*Correspondence: stefania.crispi@ibbr.cnr.it

${ }^{\dagger}$ Rossana Cuciniello and Stefania Filosa co-first authors.

${ }^{1}$ Institute of Biosciences and BioResources-UOS Naples CNR, via P.

Castellino, 111-80131 Naples, Italy

Full list of author information is available at the end of the article

\section{Background}

Cancer is a complex genetic disease mainly due to dysregulation in the expression of genes involved in critical cellular pathways. Carcinogenesis can be due to the alteration of the expression of coding sequences such as 
oncogenes or oncosuppressors, but also to the misregulation of non-coding elements whose transcription generates non-coding RNAs [1].

Non-coding RNAs for a long time after discovery were thought to be non-functional molecules and they were considered as "transcriptional noise". Subsequently, it was recognized that non-coding RNAs play a key role as transcriptional and translational regulators in different diseases including cancer [2]. This new biological role stimulated scientists in analyzing the possibility of translating their effectiveness in clinics, starting to consider them as novel drugs for the treatment of cancer and other diseases [3]. Non-coding RNAs are molecules classified according to their length: those up to 200 nucleotides long are called small non-coding RNAs (sncRNA), the others are known as long non-coding RNAs (lncRNAs) [4].

sncRNAs include microRNA (miRNAs), small interfering RNAs (siRNAs) and other small RNAs such as small nuclear RNAs (snRNAs), small nucleolar RNAs (snoRNA), Piwi-interacting RNAs (piRNAs) and transfer RNAs (tRNAs) [5].

miRNAs and siRNAs are the only sncRNAs that have been studied for therapeutics and their biology and application as anticancer will be described in this review thoroughly.

miRNAs and siRNAs are the sncRNAs widely distributed in both phylogenetic and physiological terms and are characterized by the double-stranded nature of their precursors. These sncRNAs are related in size, biogenesis and mechanism of action. miRNAs are small molecules implicated in the mechanism of RNA interference (RNAi) that performs a fine regulation of gene expression process by interfering with gene transcription thus affecting the fate of their target messenger RNAs (mRNA) that can be repressed or degraded. Similarly, siRNAs can knockdown the expression of target genes in a sequencespecific way by inducing mRNA degradation [6].

miRNAs were first described in the 1990s by Lee and colleagues that observed in C. elegans the formation of small RNAs produced by the transcription of lin-4 locus and characterized by an antisense complementarity to lin-14 gene [7]. Several years later these molecules were identified as miRNAs and since then, through cloning, sequencing and computational prediction, thousands of miRNAs were identified in different organisms. It is estimated that miRNA coding genes represent $1-5 \%$ of the mammalian genes [8].

Both miRNAs and siRNAs inhibit transcription by binding specific sequences of mRNA: miRNAs can recognize targets by perfect or imperfect complementarity; siRNAs inhibit transcription by creating a double strand molecule that binds only perfect complementary sequences on target mRNA [9]. Considering the ability of these molecules to hypothetically target the expression of any gene, starting from their discovery sncRNAs have been studied as possible tools to be used in translational and clinical approaches related in cancer and in many other diseases [10]. Although the similar activity of miRNAs and siRNAs, their application from bench to therapy are different since miRNAs are able to modulate simultaneously the expression of several different target genes, while siRNAs can specifically target a single gene at a time $[11,12]$.

Emerging therapeutic strategies based on the use of miRNAs and siRNAs are under development, with the purpose of making sncRNAs-based therapeutics a new and powerful tool to treat cancer $[13,14]$. Several studies and clinical trials have been dedicated to the development of novel anticancer treatments miRNA and siRNA-based. miRNA-based therapeutics can determine miRNA inhibition or miRNA replacement. siRNA-based therapeutics by inhibiting the expression of a specific mRNAproduce a gene silencing effect $[10,15]$.

In this review we discuss the recent progress of small RNAs-based cancer therapeutics detailing the differences between using miRNAs and siRNAs. We also report recent advances in the field that will provide valuable progress to cancer therapeutics.

Specifically, we performed a detailed literature review updated to to October 2021, searching within the main public scientific databases (Supplemental Fig. 1).

\section{Small non coding RNA biogenesis and mechanism of action}

RNA silencing is a gene regulatory system that can act either by suppressing transcription or degrading transcribed RNA. After the discovery of the first miRNA [8] it was described that exogenous double-stranded RNA (dsRNA) was able to silence gene expression through RNAi. RNAi was identified also in plants in which the silencing is concomitant with the presence of small RNAs 20-25 nucleotides long, perfectly matching to the sequence to be inactivated. Subsequent studies clarified that small RNA regulators are present in different plants and in animal species and that they can be divided into two categories: miRNAs that regulate endogenous genes and siRNAs that act to keep genome integrity against external insult of nucleic acids (viruses, transposons, transgenes) [16].

Despite their different mechanisms of action, miRNAs and siRNAs have similar physical and chemical properties, being both short RNA duplexes that target mRNAs and determine gene silencing.

miRNAs and siRNAs activity both depend upon Dicer and Argonaute (AGO), two proteins crucial for small 
RNA regulatory pathways: Dicer produces small RNAs from their double-stranded precursors, and AGO binds mature small RNAs letting them to exert gene silencing function $[17,18]$.

The modulation of endogenous or exogenous genes represents a powerful reprogrammable and tunable system to regulate gene expression.

Mature miRNAs are about 22 nucleotides long, but they are convergently transcribed from longer genes generally located in the introns of the pre-mRNA host genes $[19,20]$. Mature miRNA contains at 5 'end a 7 nucleotides sequence (seed sequence) that matches the $3^{\prime}$ UTR of mRNA target thus determining gene downregulation [21, 22] (Fig. 1).

miRNAs biogenesis takes place in the nucleus where they are transcribed by RNA Polymerase II as longer precursor (pri-miRNA) [23]. The pri-miRNA is converted into pre-miRNA $[24,25]$ and then it is translocated to the cytoplasm where it is shortened from a 70 nucleotides stem-loop structure to a 18-24 base pair double-strand RNA [26, 27]. Then two strands undergo different processes. The strand named "guide strand" is integrated in the RISC (RNA-induced silencing complex) AGO complex while the other one, the "passenger strand", is discharged from the protein complex. Theguide strand mediates gene silencing on the target mRNA by translational repression or mRNA degradation [28, 29] (Fig. 2).

The stability of base pairing of the 2-4 nucleotides at the $5^{\prime}$ end of the duplex dictates which strand becomes the guide strandand the efficacy of the silencing is dictated by the nucleotides composition of the "seed sequence" and by the degree of complementarity to the $3^{\prime}$ UTR of the target mRNA [30, 31].

Depending on the degree of complementarity, the silencing can result in either translational inhibition or in accelerating the shortening of the poly $(\mathrm{A})$ tail, thus determining a faster mRNA degradation.

The mRNAs translationally repressed by miRNA can accumulate in cytoplasmatic foci known as P-bodies (Processing-bodies) or GW-bodies (Glycine-Tryptophan bodies), twostructures containing proteins involved in mRNA decay [32].

siRNAs were first observed by transgene-induced silencing experiments in plants and it was thought that they only derived from exogenous sequences [33]. Subsequent functional studies led to detect that trans-acting siRNAs also resulted from genomic transcripts. Afterward different sources of endogenous siRNAs were identified, and it was evidenced that both exogenous and endogenous siRNAs are subjected to the same processing mechanism, with the endogenous ones having an obligate nuclear phase $[34,35]$.

siRNAs are generated from perfectly-paired dsRNAs ranging from 30 to over 100 nucleotides. They are produced in cells as response to external insults such as RNA viruses that, replicating themselves, allow the formation of dsRNA intermediates. siRNAs can also be generated from the transcription of overlapping genes, or by RNAdependent RNA polymerases that generate complementary strands from single-stranded RNA templates. The dsRNAs precursors are processed in the cytoplasm by the RNAse III Dicer, which cleaves the longer precursor

A)

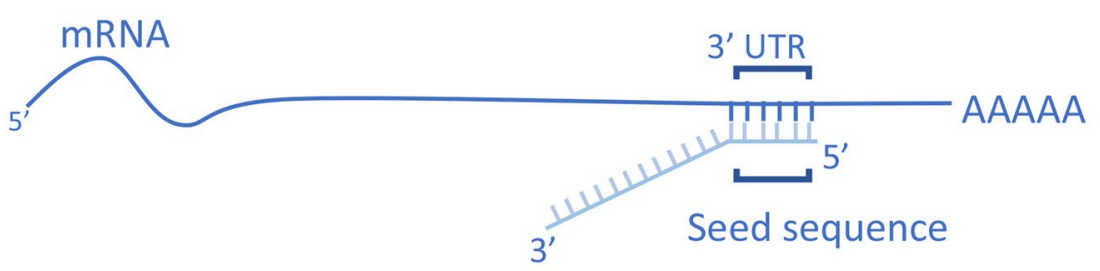

miRNA

B)

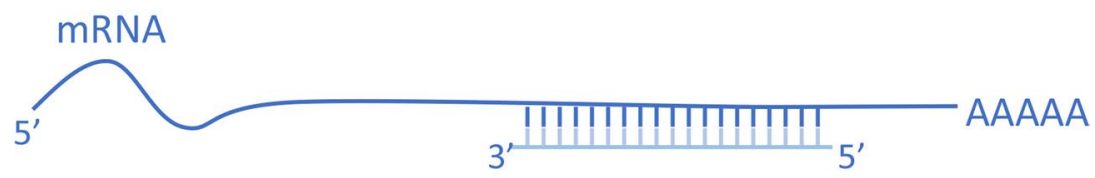

SiRNA

Fig. 1 miRNA and siRNA structures. A miRNAs are characterized by a 7 nucleotide "seed sequence" that determines gene silencing by binding the 3' UTR of mRNA target. B siRNAs bind fully complementary sequences on the mRNA target that is than degraded 


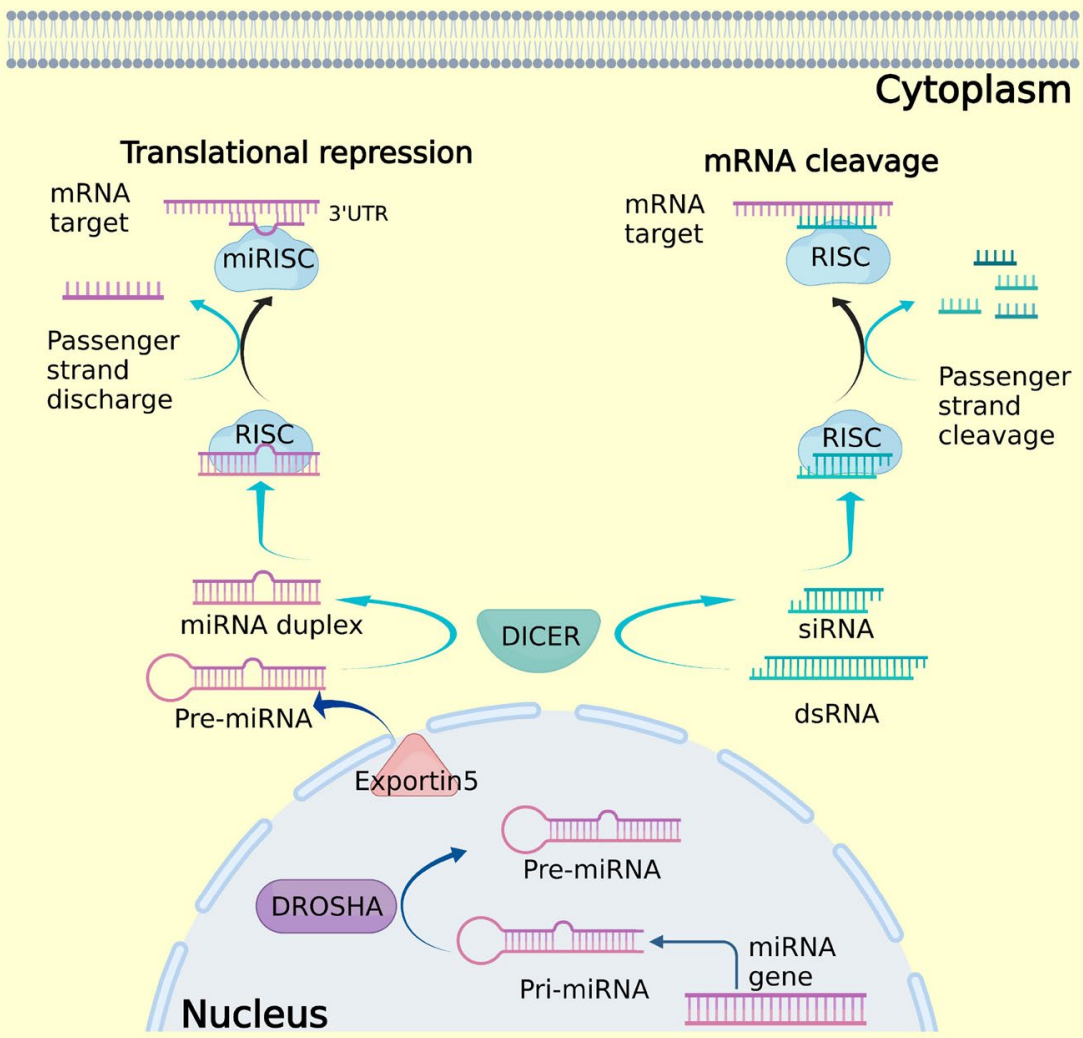

Fig. 2 miRNA and siRNA biogenesis. In the nucleus the transcribed Pri-miRNA are converted by DROSHA in Pre-miRNA. Then they are translocated in the cytoplasm by Exportin 5. In the cytoplasm, DICER cleaves the Pre-miRNA in miRNA duplex and then the RISC complex selects the guide strand with consequent RNA target repression. Exogenous dsRNAs are converted in the cytoplasm in siRNA by DICER. Then siRNA is loaded in the RISC complex and, after the cleavage of the passenger strand, the guide strand determines mRNA degradation

sequence in shorter molecules, a siRNA 21-23 nucleotides long with two nucleotides $3^{\prime}$ overhangs. The two strands of mature siRNAs are then processed by the RISC complex. In this case, and differently from miRNAs maturation, the passenger strand is the sense strand while the antisense strand always represents the guide strand. dsRNAs bind and activate the RISC complex, and then AGO cleaves the passenger strand retaining the guide strand. The assembled RISC-siRNA complex is now ready totarget specific mRNAs with binding occurring between fully complementary sequences (Fig. 2). Subsequently, the endonuclease Slicer in the RISC effector complex cleaves the mRNA sequence complementary to the siRNA guide. The mRNA is then degraded by exonucleases and thus silenced [36-39].

miRNAs can modulate gene expression inducing translational repression, mRNA deadenylation or decapping but they can also activate transcription or translation [40].
In most cases, miRNAs induce mRNA degradation or translational repression interacting with the $3^{\prime}$ UTR of target mRNAs [41]. In addition, also the binding to $5^{\prime}$ UTR or to the gene coding sequence induces gene silencing $[42,43]$. In contrast, the interaction of miRNAs with promoter regions has been reported to activate transcription [44].

As reported before the recognition between miRNAs and mRNAs does not require perfect pairing. For this reason, a miRNA can recognize and down-regulate the expression of different mRNAs. Interestingly, an incomplete base pairing between miRNA and mRNA, does not activate AGO of the RISC complex, but miRNA can mediate silencing through translational repression, or by degradation, deadenylation, decapping or exonuclease action. Of course, a perfect pairing of miRNA with mRNA generates a double strand RNA recognized by AGO that leads to endonucleolytic cleavage of mRNA [45]. 
Unlike miRNAs, siRNAs only determine long-term silencing of the mRNAs encoding genes. siRNA by binding only to specific mRNA targets, generates a perfect pairing double strand RNA that is cleaved by AGO [46].

\section{Strategies for sncRNA-based gene targeting in therapeutics}

The incidence of cancer is rapidly increasing thus resulting in a high economic and social impact [47]. Conventional cancer treatments, such as removal of cancer tissues and metastases with surgery as well as the use of chemotherapeutics or radiotherapy, improved overall survival but, nevertheless, they fail to prevent cancer recurrence and metastasis [48-50]. Furthermore, chemotherapy determines serious adverse effects such as systemic toxicity and increases the multiple drug resistance, all issues requiring the development of new more effective therapeutic strategies. Trying to improve cancer therapeutics, emerging strategies based on sncRNAs molecules are under development, with the purpose of making miRNA and siRNA-based drugs as new and powerful tools to cure cancer [14].

Undesired expression of mutated genes or overexpression of certain genes can be the origin of different diseases including cancer. The major limitation of chemical drugs is that they act only on specific proteins or enzymes. In contrast, miRNAs and siRNAs due to their ability to modulate gene expression are able to act also on "non-druggable" targets and have the huge potential to be considered as therapeutic agents. For example, gene dysregulation that characterizes cancer cells can be restored using miRNA replacement therapy or miRNA inhibitors, with the aim to restore their physiological expression.

\section{miRNAs as therapeutic agents}

Considering that the majority of human genes contain at least one miRNA consensus site, miRNAs could be used in different therapeutic applications [51]. Two main strategies can be used to manipulate gene expression through miRNAs, depending on whether miRNA should be reintroduced (mimic) or downregulated (inhibitor) to modulate the amount of mRNA target in the cell.

miRNA mimics are synthetic double-stranded oligonucleotides that overexpress the target miRNA sequences. They are specifically designed to achieve the same biological functions of the endogenous miRNAs resulting in downregulation of cancer cells. Ectopical expression of miRNAs could be done using vectors that overexpress the target miRNA or using miRNA mimics. In both methods, the reintroduced miRNA achieves the same biological functions of the endogenous miRNA, by silencing the target mRNA (Fig. $3 \mathrm{~A}$ ). To exert this function, the synthetic miRNA should have a structure able to be loaded in the RISC complex, to operate as a guide strand and to recognize and then interfere with the mRNA targets. Thus, miRNA mimics design should be performed considering the chemical modification needed to improve their binding affinity, biostability and pharmacokinetic properties. For example, double-stranded molecules composed by both passenger and guide strand resulted in a better silencing effect, due to a more efficient loading of the RNA molecule in the RISC complex. Synthetic single stranded miRNAs are less suitable since they are rapidly degraded in biological fluids, and are characterized by a short half-life after administration [52-54].

As opposed to miRNA mimics, miRNA inhibitors are designed to specifically block the upregulated expression of miRNAs associated with cancer development. The most commonly used method for targeting specific miRNAs is based on the generation of antisense oligonucleotides (ASOs), or antagomir, that specifically bind the endogenous target miRNA inhibiting its function. ASOs are designed as a single-stranded structure complementary to the sense strand of the target miRNA (Fig. 3 B). They exert their function as competitive inhibitors $[55,56]$. In this case it is possible to design molecules with higher affinity to miRNAs, by inserting specific modifications such as the insertion of several bicyclic RNA analogues that form a "locked" conformation thus ensuring a better hybridization in miRNAs targeting process. These molecules are known as Locked nucleic acids (LNAs) $[57,58]$. A different structural modification, based on the development of heteroduplex oligonucleotides anti-miRNA, also shows improved suppression efficiency [59]. Another strategy to increase the targeting efficacy is based on the development of peptide nucleic acids (PNA) that specifically target full-length miRNAs by Watson-Crick recognition (Fig. $3 \mathrm{C}$ ). These structures are relatively stable since they are synthetic nucleic acid analogues that possess a neutral backbone and are resistant to enzymatic degradation [60]. Other powerful competitive miRNAs inhibitors are the miRNA sponges, that are transcribed from strong promoters and contain multiple tandem binding sites to target a miRNA of interest (Fig. 3 E). In the cells, miRNA sponges, competing with the native targets of miRNAs, allow increased expression of the mRNAs target. miRNA sponges are longer nucleic acids, expressed by transgenic vectors, that specifically inhibit miRNA with a complementary heptameric seed. Their peculiar structure allows inhibition of a single miRNA or of a miRNA family whose heptameric seed binding sequence is the same, allowing increased expression of all mRNAs that are modulated by inhibited miRNA. Using appropriate promoters, they should work in any kind of cell or transgenic model organism 


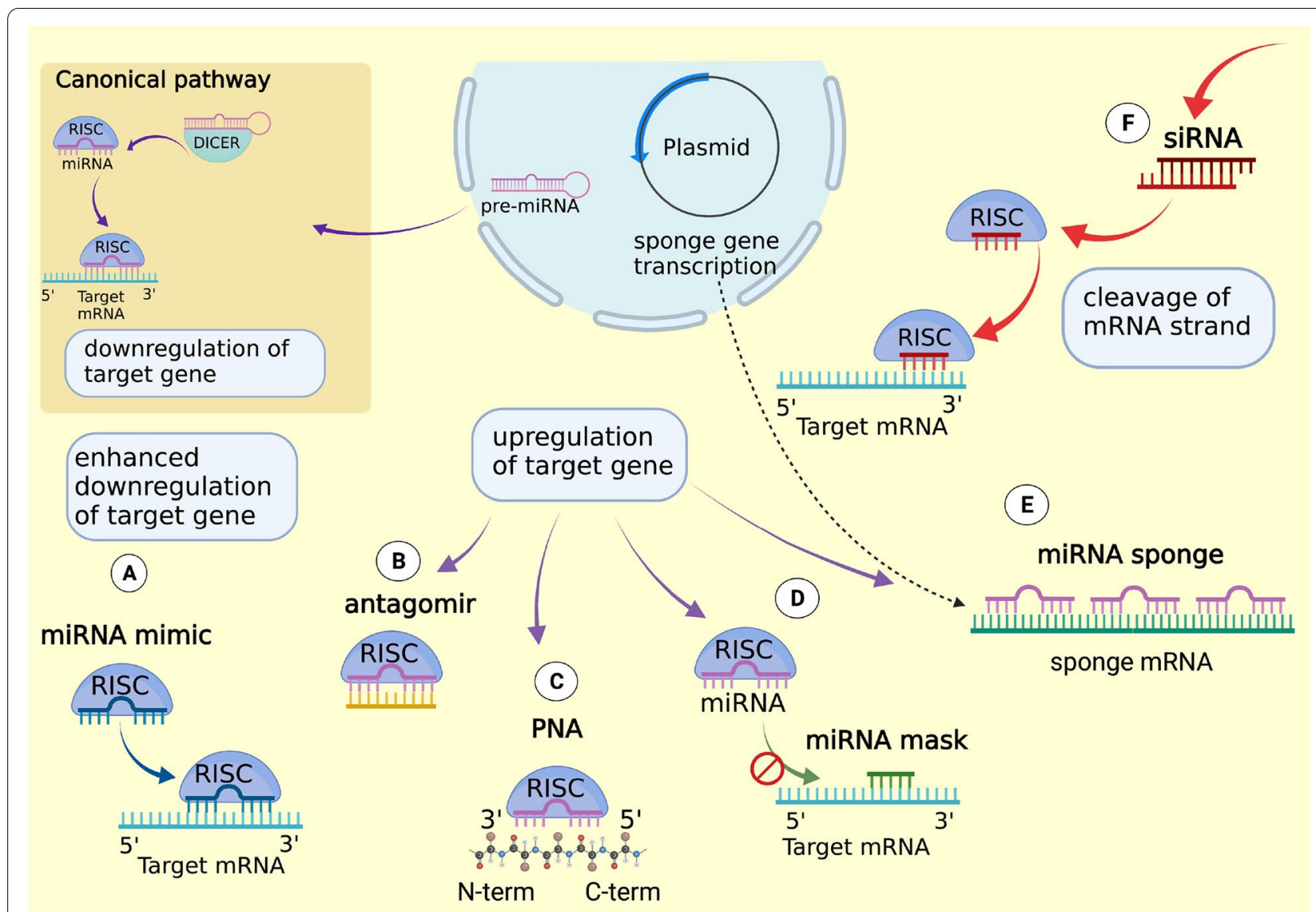

Fig. 3 Schematic description of the strategies used to manipulate gene expression by sncRNAs: A) miRNA mimics; B) miRNA antagomirs; C) PNAs D) miRNA masks $\mathbf{E}$ ) miRNA sponges and $\mathbf{F}$ ) siRNAs

[61]. The main advantage of sponges is that the use of transgene overcomes the problems related to the oligonucleotides uptake [62]. However, a limitation of this approach is that sponges need to be administered in higher concentrations than ASOs, thus increasing the possible off targets.

The miRNA-mask is a different strategy able to silence the target gene by competing with the binding of miRNA that regulates that gene on the $3^{\prime}$ UTR site. To be effective, the miRNA-mask should contain the recognition motif for a miRNA within the $3^{\prime}$ UTR of the target gene and the sequences containing the miRNAbinding motif. The presence of this stretch is fundamental to achieve gene specificity of miRNA-mask action allowing miRNA-mask binding.

The binding of miRNA-mask to the binding site of miRNAs in $3^{\prime}$ UTR of the target mRNA avoids the recognition of miRNA on the target mRNA and the consequent degradation by RISC complex, leading to a relief of translational repression without affecting miRNA levels (Fig. 3 D) [63].

\section{siRNAs as therapeutic agents}

siRNAs are double strands molecules containing 19-21 nucleotides with two nucleotide overhangs at the $3^{\prime}$ end, usually TT and UU, that allow them to be recognized by the RNAi machinery. In vitro studies have demonstrated that longer dsRNAs with 27 nucleotides are more efficient. This feature could be related to the fact that longer structures are more easily processed by Dicer allowing a better gene silencing activity [64]. It has also been demonstrated that the siRNAs for therapeutic applications should not be longer than 30 nucleotides to avoid an immune response through the activation of Interferon pathway [65].

siRNAs can exert RNA interference in a very specific manner by binding the target mRNAs with a complete sequence base pairing (Fig. 1). As for miRNAs, 
therapeutic effectiveness of siRNAs strongly depends on the molecule design.

A critical step is the selection of the mRNA sequence that needs to be recognized by siRNA. In fact, to achieve efficient and specific gene silencing it is crucial to select the optimal sequence, excluding potential off targets. Even if this problem can be addressed by using specific different prediction algorithms emerged in recent years, the siRNA efficacy should be experimentally validated $[66,67]$.

Another important issue to consider in siRNA therapeutics is that an incorrect orientation of the dsRNA could determine an incorrect selection of the guide strand determining the silencing of non-intended mRNA. As described before, the RNA interference proceeds, after the correct loading of the siRNA in the AGO/RISC complex, with the discard of the passenger strand and the use of the guide strand for the mRNA degradation (Fig. 3 F) [68]. The guide strand is selected according to the loading orientation of the molecule in the AGO protein and both strands could be selected as guide strand. Therefore, the dsRNA to be used as siRNAs needs to be designed to assure proper strand selection by the RISC complex. This can be carried out considering two parameters: the asymmetry rule and the $5^{\prime}$ nucleotide preference.

The asymmetry rule is based on the finding that the thermostability of the two ends of the duplex contributes to the selection of the guide strand [30]. Consequently, the strand with a $5^{\prime}$ end that contains higher $\mathrm{A} / \mathrm{U}$ content would be discarded due to its high stability. A correct loading of the dsRNA in the RNAi machinery will be achieved by designing a nucleotide sequence with lower stability that will be selected as guide strand.

The $5^{\prime}$ nucleotide preference parameter is based on the specificity of AGO proteins toward a strand with a $U$ or an $\mathrm{A}$ at the $5^{\prime}$ end that is selected as a guide strand. Thus, the passenger strand of the duplex should contain $C$ and $\mathrm{G}$ to reduce the possibility of an incorrect selection of the guide strand [69].

Finally, also the nucleotide composition of siRNAs can affect the silencing efficacy. For example, the G/C content of siRNAs could affect the thermodynamic stability and the accessibility to the target site. siRNAs with very high content of G/C have been described to show reduced efficiency, while siRNAs with a content of G/C between 30 and $64 \%$ exert an efficient gene silencing effect [70, 71].

To manipulate gene expression through siRNAs it is essential to design a specific oligonucleotide sequence that perfectly matches with mRNA target to minimize the off-target effects.

Off-target effect can occur either in presence of high concentration of siRNA or when there is amiRNA-like off-target effect [72, 73]. Excessive siRNAs concentration, competing with the same protein machinery used by endogenous miRNAs, can cause off-target effect through the saturation of the RNAi machinery [74]. The miRNAlike off-target effect occurs when a siRNA induces a sequence-dependent regulation of unintended transcripts through the sequence complementarity to their $3^{\prime}$ UTRs. This can determine mRNA degradation mediated by partial sequence complementation thus resulting in a miRNA-like translational inhibition with a decrease of the protein level [75]. In particular, it happens if the design of the $5^{\prime}$ of the guide strand is not accurate or if the siRNA is totally or partially complementary to the $3^{\prime}$ UTR of the mRNA [72, 76, 77].

A common approach to resolve both events that determine off-targets is the use of the minimum possible concentration of siRNA, as the previous mechanisms are dependent from the concentration [74]. Another possibility is to use low concentrations of multiple siRNAs sequences targeting the same mRNA that, recognizing different off-targets, will reduce the risk of the off-target effect [69]. The miRNA-likeoff targeteffect could be also overcome by avoiding in the siRNA design the seed sequence of miRNAs or the 2-7 nucleotides of $5^{\prime}$ end, identifiable with the help of miRNA databases [72, 76, 77].

\section{sncRNA-based therapeutics in oncology}

There is a growing interest of the pharma companies in identifying new molecules to be used as novel drugs against cancer. In this regard, sncRNAs have the potential to become a new class of drugs active in different diseases including cancer.

The recent advances in the identification of the molecular pathways involved in cancer onset and progression open new possibilities for cancer therapy. In this regard the identification of the sncRNA targets represent a valuable tool for gene silencing, enabling the suppression of oncogeneic factors. Recently, extensive analyses have been performed on the identification of novel strategies to specifically target oncogene and tumor suppressor genes. For example, many studies are reported for prostate cancer, the second most commonly occurring cancer and one of the leading causes of death in man. Recent studies led to identify sncRNAs able to inhibit target genes involved in this cancer pathogenesis [78]. Interestingly, most of thesesncRNAs have been tested on ongoing preclinicalstudies to unravel the efficacy of sncRNAs-based therapeutics (Supplemental Table 1).

Despite the large number of sncRNAs analysed as therapeutics, only 3 miRNAs (Table 1 ) and 10 siRNAs (Table 2) are currently in clinical trials as anticancer and they will be described below [79]. 
Table 1 miRNAs in cancer therapeutics

\begin{tabular}{lllll}
\hline Target & Drug name & Cancer & Phase & $\begin{array}{l}\text { ClinicalTrials. } \\
\text { gov Identifier }\end{array}$ \\
\hline Mir 16 & TargomiRs & Malignant pleura mesothelioma, Non small-cell lung cancer & I & NCT02369198 \\
Mir 155 & MRG 106 & Lymphomas, Leukemia & NCT02580552 \\
Mir 34a & MRX34 & Melanoma, Primary liver cancer; Hematologic malignancies & I Terminated & NCT01829971 \\
Mir 34a & MRX34 & Melanoma, Primary liver cancer; Hematologic malignancies & I/II Withdrawn & NCT02862145 \\
\hline
\end{tabular}

Table 2 siRNAs in cancer therapeutics

\begin{tabular}{|c|c|c|c|c|}
\hline Target & Drug name & Cancer & Phase & $\begin{array}{l}\text { ClinicalTrials. } \\
\text { gov Identifier }\end{array}$ \\
\hline PKN3 & Atu027 & Carcinoma, Pancreatic Ductal & 1 & NCT00938574 \\
\hline PKN3 & Atu027 & Carcinoma, Pancreatic Ductal & $|/| \mid$ & NCT01808638 \\
\hline KRAS & siG12D LODER & Pancreatic ductal adenocarcinoma, pancreatic cancer & 1 & NCT01188785 \\
\hline KRAS & siG12D LODER & Pancreatic ductal adenocarcinoma, pancreatic cancer & $\|$ & NCT01676259 \\
\hline KrasG12D mutation & $\begin{array}{l}\text { Mesenchymal stromal cells- } \\
\text { derived exosomes with KRAS } \\
\text { G12D siRNA }\end{array}$ & Pancreatic cancer & I & NCT03608631 \\
\hline PLK1 & TKM-080301 & $\begin{array}{l}\text { Adrenal cortical carcinoma, neuroendocrine tumor, hepa- } \\
\text { tocellular carcinoma }\end{array}$ & $|/| \mid$ & NCT01262235 \\
\hline PLK1 & TKM-080301 & $\begin{array}{l}\text { Adrenal cortical carcinoma, neuroendocrine tumor, hepa- } \\
\text { tocellular carcinoma }\end{array}$ & l & NCT01437007 \\
\hline PLK1 & TKM-080301 & $\begin{array}{l}\text { Adrenal cortical carcinoma, neuroendocrine tumor, hepa- } \\
\text { tocellular carcinoma }\end{array}$ & $|/| \mid$ & NCT02191878 \\
\hline AR V7 variant & SXL01 & Metastatic castration-resistant prostate cancer (CRPC) & । & NCT02866916 \\
\hline EphA2 & EPHARNA & Advanced Malignant Solid Neoplasm & । & NCT01591356 \\
\hline $\mathrm{BCL} 2 \mathrm{~L} 12$ & NU-0129 & Gliosarcoma, recurrent Glioblastoma & l & NCT03020017 \\
\hline VEGF and KSP & ALN-VSPO2 & Solid tumors & I & NCT01158079 \\
\hline RRM2 & CALAA-01 & Cancer, solid tumor & I Terminated & NCT00689065 \\
\hline MYC & DCR-MYC & $\begin{array}{l}\text { Hepatocellular Carcinoma, Solid Tumors; Multiple } \\
\text { Myeloma, or Lymphoma }\end{array}$ & I Terminated & NCT02314052 \\
\hline MYC & DCR-MYC & $\begin{array}{l}\text { Hepatocellular Carcinoma, Solid Tumors; Multiple } \\
\text { Myeloma, or Lymphoma }\end{array}$ & Ib/II Terminated & NCT02110563 \\
\hline
\end{tabular}

\section{miRNAs in clinical trials}

TargomiRs One of the first studies demonstrating an involvement of miRNA dysregulation in cancer reported that the loss of the miR-15a/16-1 resulted in chronic lymphocytic leukemia (CLL). miR-15a/16-1 was the first miRNA discovered to function as tumor suppressor by directly targeting BCL2 gene that inhibits apoptosis and that is one of the most important oncogenes involved in lymphoma development [80]. Two different mice carrying miR-15/16 deletion have been generated and both lines developed lymphomas. Although these mice showed only mild upregulation of BCL2 expression, a robust upregulation of several predicted miR-15/16 targets such as CCND1, CCND2, and IGF1R1 has been observed [81].
To analyse the anticancer effect miR-15/16 ectopic expression it was developed miR-16 mimic (TargomiRs) that was tested in a phase I clinical trial (NCT02369198) in patients with Malignant pleural mesothelioma or advanced non-small cell lung cancer. Results provided data that confirmed the activity of TargomiRs as inhibitor of tumor growth indicating that restoring the level of miRNAs that act as oncosuppressor can represent a very attractive way to inhibit tumor growth. The phase II has been planned to compare the TargomiRs effects with those obtained in patients after the second and third cycle of chemotherapy [82].

MRG-106 miR-155 is an oncogenic miRNA overexpressed both in hematological and in solid tumors and it is known to be a "bridge between inflammation and 
cancer" [83]. miR-155 is used as a diagnostic tool to distinguish between benign and malignant inflammation in cutaneous T-cell lymphoma (CTCL) since it is overexpressed in the skin of CTCL patients. miR-155 is a transcriptional target of STAT5 and upregulation of STAT5/ miR-155 pathway has been described to be involved in proliferation of malignant $\mathrm{T}$ cells and it is also known that the antisense miR-155 inhibits the process. For this reason, miR-155 has been considered as a putative target for therapy in CTCL [84]. In vivo trials have been performed using an oligonucleotide inhibitor of miR-155 (MRG-106), which was able to activate the specific miR155 targets. Interestingly, MRG-106 was shown to have a significant pharmacodynamic activity in the preclinical model and a phase I clinical trial (NCT03837457) evaluated its safety, tolerability, pharmacokinetics and preliminary efficacy. The preliminary results showed that MRG106 was well-tolerated and the efficacy in the recovery of cutaneous lesions prompted additional therapeutic analysis [85].

MRX34 miR-34a is a tumor suppressor that is lost or under-expressed in different tumor types. Retrospective clinical studies reported a negative correlation between survival and reduction of miR-34a expression [86]. miR34a downregulated the expression of many oncogenes, including MET, MYC, BCL2, PD-L1. In vitro studies showed that reintroduction of a miR-34a mimetic in tumor cell lines reduced cell proliferation, migration and invasion. In vivo, preclinical studies in animal models showed that miR-34a inhibited primary tumor growth, blocked metastasis, and improved survival. The effectiveness of miR-34a mimetic (MRX34) was then confirmed in mouse models of hepatocellular carcinoma [87].

Prompted by encouraging preclinical data, two phase I trials (NCT01829971 and NCT02862145) have been initiated to evaluate MRX34 safety, pharmacokinetics and clinical activity. The NCT01829971 recruited adult patients suffering from refractory advanced solid tumors or hematologic malignancies, the NCT02862145 enrolled patients with melanoma. The results of the first trial evidenced the anticancer activity and confirmed that miR34a mimetic was able to modulate the expression of its targets. Unfortunately, both studies have been stopped due to a severe immune-mediated response [88].

\section{siRNAs in clinical trials}

Atu027

Protein kinase N3 (PKN3) is a downstream effector of the PI3K-signal transduction pathway. This pathway is implicated in the control of morphology and locomotion of endothelial and cancer cells. PKN3 has been considered a promising therapeutic target to inhibit metastasis formation. Indeed, gene silencing of PKN by siRNA (Atu027) in vascular and lymphatic endothelial cells has been reported to inhibit tumor growth and lymph node metastasis formation in mouse models [89].

A phase I clinical trial (NCT00938574) demonstrated that Atu027 is well tolerated and has antitumor activity [90]. A subsequent phase I/II study (NCT01808638) has been started to evaluate the safety and activity of Atu027 in combination with the standard chemotherapeutic gentamicin, as a new treatment strategy for advanced pancreatic cancer disease. This trial confirmed the efficacy of Atu027 and confirmed the importance of continuing the study with the aim to use this molecule as standard drug for the treatment of advanced pancreatic carcinoma [91].

\section{SIG12D LODER}

KRAS is a member of the small GTPase superfamily and mutated KRAS is considered a hallmark of pancreatic cancer. Suppression of this oncogene by RNAi was reported to inhibit growth both in vitro and in vivo [92, 93].

Since most pancreatic ductal adenocarcinomas are caused by KRAS G12D mutation, it has been developed a biodegradable matrix to deliver KRAS siRNA-G12D (siG12D LODER). The ability of this drug to silence the upregulated mutated gene and determine apoptosis of cancer cells, slowing the tumor growth, has been assessed and confirmed in an orthotopic mouse model [94].

A phase I clinical trial (NCT01188785) demonstrated that siG12D LODER is well tolerated, safe and has potential efficacy against pancreatic ductal adenocarcinomas [95]. In addition, a phase II (NCT01676259) continues to evaluate the efficacy of siG12D LODER in combination with chemotherapy. The patients have been enrolled, but, to date, the results have not been reported yet [96].

\section{Mesenchymal stromal cells-derived exosomes loaded with KRAS G12D siRNA}

Another phase I trial (NCT03608631) to try silencing the oncogene KRAS G12S is recruiting patients with metastatic pancreatic cancer. The aim of the study is to identify the best dose with reduced side effects of Mesenchymal Stromal Cells-derived Exosomes loaded with KRAS G12D siRNA [97]. In particular, patients affected by pancreatic ductal adenocarcinoma that overexpress KrasG12D mutation will be recruited. The treatment will evaluate the efficacy of the silencing and other parameters such as disease control rate and the median overall survival. For this trial the last update has been reported in April 2021 and the estimated study completion date will be March 2022. 


\section{TKM-080301}

Polo-like kinases (PLKs) is a family of proteins, composed of at least 5 members, that has an important role in maintenance of mitotic integrity. Among them, PLK1 is the kinase involved in the control of mitotic entry, centrosome maturation, bipolar spindle formation, cohesion dissociation, chromosome congression and segregation, and cytokinesis PLK is over expressed in many types of tumors and its expression has been correlated with poor diagnosis.

Knockdown of PLK1 expression, using siRNA, induced a reduction in cell proliferation in different hepatocellular carcinoma cell [97]. Therefore, PLK1 was used as target for cancer treatment. Three phase I/II different clinical trials have been registered (NCT01262235 NCT01437007 NCT02191878) and all of them will evaluate the safety, pharmacokinetics and preliminary antitumor activity of siRNA against PLK1 (TKM-080301). TKM-080301 showed a favorable toxicity profile and preliminary anti-tumor efficacy has been observed. However,the clinical trials did not demonstrate improved survival in patientsand did not support further evaluation as a single agent [98].

\section{SXL01 (PROSTIRNA)}

Prostate cancers are the second leading cause of cancer death in men in the world. Hormone- resistant disease is characterized by overexpression of Androgen receptor (AR). These tumors do not respond to hormonal ablation and additionally, this practice often results in more aggressive cancer relapse.

It has been demonstrated that knocking down the androgen receptor by siRNA leads to significant apoptotic cell death, inhibiting the Bcl-xL-mediated survival signal that acts downstream of androgen receptor-dependent survival pathway [99].

In vivo, the efficacy of androgen receptor silencing has been tested in mouse models of prostate cancer. The results obtained suggested that this strategy efficiently knocks down androgen receptors and could be considered a new therapeutic approach for these cancers [100].

A phase I clinical trial (NCT02866916) has been carried out in humans using a siRNA, called SXL01(PROSTIRNA), to prevent the synthesis of the androgen receptor. The main goal of this trial is to evaluate the safety, the tolerability and the therapeutic effects of SXL01 in patients suffering from castration-resistant prostate carcinomas. Results have not been published until now. Therefore, in January 2021 the recruitment was withdrawn and the study cancelled.

\section{EPHARNA}

EphA2 is a tyrosine kinases receptor member of a largest subfamily composed of 14 receptors and 8 ligands. EphA2 is overexpressed in different cancers including breast, endometrial, lung, ovarian, pancreatic and prostate, and its expression is always associated with adverse outcomes. EphA2 acts as an oncoprotein influencing cell proliferation, survival, migration, invasion, and angiogenesis; moreover, it has been reported in preclinical studies that its down-regulation reduces tumorigenicity [101, 102]. All these data suggest that EphA2 is an ideal therapeutic target.

In vitro and in vivo studies demonstrated that EPHARNA, the EphA2-siRNA, reduces tumor growth dramatically and acts as an anti-angiogenic. Moreover, analysis in Rhesus macaques demonstrated that EPHARNA is well tolerated at all tested doses [103].

EPHARNA is currently used in a phase I clinical trial (NCT01591356) to study, also in patients with advanced metastatic solid cancer, the safety, the maximal tolerated dose and to determine its efficacy on tumor growth. The trial is ongoing and no results have been posted yet [104].

\section{Nu-0129}

Bcl2L12 is a member of the Bcl2 family, containing a Bcl-2 homology domain 2 (BH2). It has been described to have anti-apoptotic properties, but this role remains controversial in different cancer types. Although the fulllength mRNA transcript of Bcl2L12 is expressed in many tissues, its overexpression in most human glioblastomas has been associated with tumor cell progression and tumor cell resistance to apoptosis. Conversely, knockdown of Bcl2L12 both in astrocytes and glioma cell lines resulted in enhanced apoptosis [105].

It has been registered an early phase I clinical trial (NCT03020017) to evaluate, in patients affected by recurrent glioblastoma multiforme or gliosarcoma, the safety of a drug called NU-0129. This drug should be able to target Bcl2L12 and stop cancer cells from growing.

NU-0129 is based on spherical nucleic acids (SNAs), and in particular consists of gold nanoparticle cores covalently conjugated with radially oriented and densely packed siRNA Bcl2L12 oligonucleotides. This novel nanotherapeutic is able to cross the blood brain barrier and the nucleic acid component is able to target Bcl2L12 allowing the induction of apoptosis.

Preliminary results showed that NU-0129 was well tolerated in glioblastoma patients with no adverse effects. There was also evidence that it is able to cross the blood brain barrier. Bcl2L12 expression and apoptotic markers analysis are pending [106]. 


\section{ALN-VSP02}

ALN-VSP02 is a RNAi-based therapeutic targeting the expression of vascular endothelial growth factor (VEGF)$\mathrm{A}$ and kinesin spindle protein (KSP). VEGF-A is an angiogenic factor that promotes tumor-associated angiogenesis, inducing proliferation and migration of vascular endothelial cells. KSP plays an essential role in mitosis mediating centrosome separation, assembly and maintenance of mitotic spindle. Inhibition of KSR results in mitotic arrest and, ultimately, in apoptosis. Both VEGF-A and KSR are overexpressed in different types of tumors and different inhibitors of both proteins are under study as novel anticancer therapeutics.

In a phase I clinical trial (NCT00882180) the safety, tolerability, pharmacokinetics, and pharmacodynamics and the ability to reduce expression of VEGF-A and KSR of ALN-VSP02 was analysed in patients with advanced solid tumors with liver involvement. The results of the study showed that ALN-VSP02 is well tolerated, that the drug was delivered to the tumor and that VEGF mRNA was downregulated in a liver and extra hepatic metastasis. An extension of this phase I study (NCT01158079) has been started to analyse over the time the therapeutic efficacy of ALN-VSP02in patients who had clinical benefit as stable disease or better. The next phase II study will enrol patients treated with the established active dosage [107].

\section{CALAA-01}

Ribonucleotide reductase (RNR) is a ubiquitous rate-limiting enzyme that catalyzes de novo formation of deoxyribonucleotides. It is required for DNA synthesis and repair, and for maintaining a balanced dNTP pool. RNR is a tetrameric protein formed by two kinds of subunits: the large ribonucleotide reductase subunit M1 and two different small subunits RRM2 and RRM2B. High expression of RRM2 is common in cancers including melanoma where it influences survival, proliferation, apoptosis, and chemoresistance [108]. For this reason, RRM2 is a cancer therapeutic target.

CALAA-01 is a siRNA-based therapeutic interfering RRM2 that is able to recognize tumor cells expressing transferrin receptors. In phase I clinical trial (NCT00689065) CALAA-01 was well tolerated during the initial dose escalation. Furthermore, tumor biopsy from patients with metastatic melanoma showed that nanoparticles were localized in the tumor cells, but not in the surrounding normal epidermis and that RRM2 mRNA and protein levels were reduced [109].

\section{$D C R-M Y C$}

C-Myc is a multifunctional transcription factor considered to be a "master regulator" of cellular metabolism and proliferation. Due to its important role as "primary oncoprotein" regulating many aspects of tumorigenesis, it represents a unique opportunity to develop novel cancer therapies [110].

DCR-MYC is a siRNA that specifically targets the oncogene MYC.

In a phase I clinical trial (NCT02110563) the safety in a dose-escalation study, the pharmacokinetics, the pharmacodynamics and clinical activity of DCR-MYC were analyzed in patients with advanced solid tumors, multiple myeloma or lymphoma in advanced solid tumors and hematological malignancies. The study demonstrated that DCR-MYC is well tolerated and shows promising initial clinical and metabolic responses [111]. In a phase Ib/II clinical trial (NCT02314052) the safety and tolerability ability to inhibit MYC of DCR-MYC were evaluated in patients with advanced hepatocellular carcinoma. Although the result of the phase I study supported the validation of MYC as a therapeutic target, both studies have been "Terminated" due to sponsor decision.

\section{siRNA -based cancer immunotherapy}

Recent studies describe the use of specific siRNAs to develop specific cancer vaccines. These methods are based on the ability of siRNAs to produce a large number of antigens thus triggering a strong and specific immune response. In addition, cancer tumor antigenic proteins expressed in the cytoplasm of antigen presenting cells are able to induce a cytotoxic $\mathrm{T}$ cell response. This process involves the formation of a protein complex between MHC class I and peptide epitopes [112].

Ongoing cancer clinical trials based on siRNAs immunotherapy have been integrated with cell-based immunotherapy. In fact, in this way the immunogenicity of immune cells such as dendritic cells or $\mathrm{T}$ cells can be restored or enhanced. These immune cells are transfected ex-vivo with tumor-associated antigen encoding siRNAs through electroporation or nanoparticle approaches. Then the transfected cells are re-infused into the patient where they determine cancer cells death.

Different therapies developed in this way and that are undergoing phase I/II clinical trials are based on the use of siRNAs transfected dendritic cells [113].

Three siRNA-based immunotherapies are ongoing in clinical trial:

\section{PSCT19}

A phase I/II study (NCT02528682) used for hematological malignancies is based on siRNA immunotherapy by silencing programmed death-protein 1 (PD-1) and its ligands 1 and 2 (PD-L1/2). Their inhibition enhances $\mathrm{T}$ cell immune responses against tumor cells [114]. This method has been used also for solid tumor treatment. 


\section{iPsiRNA}

A phase I clinical trial (NCT00672542) based on siRNA targeting immunoproteasome (iPsiRNA) subunits has been started to treat metastatic melanoma. The therapy is based on the different composition of the intracellular proteasomes in normal cells and in cancer cells. In fact, after exposure to inflammatory mediators normal cells change the proteasome from constitutive proteasome to immunoproteasome while cancer cells fail to express immunoproteasome. By modulating the proteasome of the mature dendritic cells, it is possible to stimulate a specific $\mathrm{T}$ cell response against melanoma cells. In this study, dendritic cells transfected with RNA encoding melanoma tumor-associated antigen stimulating a $\mathrm{T}$ cell response will be appropriately directed against melanoma cells [115].

\section{APN401}

A recent study based on the inhibition of Casitas-B-lineage lymphoma protein-b, an intracellular checkpoint limiting lymphocyte activation, has been initiated to treat different solid tumors. Previous studies in mouse models reported that the inhibition of this protein enhances the antitumor activity mediated by $\mathrm{T}$ cell and natural killer cell. APN401 is autologous cellular therapy consisting of peripheral blood mononuclear cells silenced for Casitas-B-lineage lymphoma protein-b. A first phase I clinical trial (NCT02166255) used a single intravenous infusion suspension of APN401 to treat patients with solid tumors; the results showed that APN401 increased cytokine production [116] and supported a second phase I clinical trial (NCT03087591) in which the effect of multiple infusions is tested [117].

\section{Delivery strategies for miRNA and siRNA therapeutics}

The availability of small non-coding RNAs as cancer therapeutics represents a novel strategy to overcome pharmacological barriers involved in therapeutic resistance. The advantage in using sncRNAs in cancer therapy is that these molecules, affecting multiple signalling pathways, result more effectively in the majority of standard chemotherapeutics that usually target a single gene.

The major limitation in sncRNAs-based therapeutics is due to the difficulty in tuning their expression in cancer cells and also to the efficacy of their delivery into cells. Both these aspects should be taken into account when it is needed to translate to the clinic the use of sncRNAs molecules.

To be efficient a delivery system must guarantee that miRNAs and siRNAs reach their site of action, and avoid their degradation by nucleases present in biological fluids. Since miRNAs and siRNAs show similar chemical properties and exploit the same protein machinery to exert the gene silencing effect, similar delivery technologies can be developed for both molecules (Fig. 4).

As detailed above, the overexpression of a specific target miRNA can be carried out by using expression vectors or miRNA mimics and gene silencing can be realized by designing specific siRNAs.

miRNAs and siRNAs after entering into cells are converted into active, single-stranded molecules, but different limitations can result in poor effectiveness. For instance, the loss of RNA backbone modifications can prevent synthetic sncRNAs to reach their target location; they also can be degraded by nucleases present in the biological systems or can be poorly uptaken through cell membranes. To overcome these difficulties chemical modifications specific for each target tissue have been designed to optimize RNA oligonucleotide stability [118]. miRNAs and siRNAs both are characterized by low cellular uptake and can be easily degraded by nucleasemediated degradation. Therefore, to have efficient delivery into target cells they should be delivered using proper carrier systems. Different delivery systems have been developed based on the use of viral systems, polymers, liposomes, and nanoparticles. In addition, the use of either lipids or exosomes greatly increases the uptake of synthetic sncRNAs.

The use of viral vectors to deliver sncRNAs is one of the first delivery systems developed. It is based on the use of viral vectors that show high transduction efficiency. In fact, RNA-encoding viruses are efficiently transferred in the nucleus of mammalian cells by adeno-associated viruses, adenoviruses and lentiviruses determining gene silencing [119]. Viral vectors have the advantage to efficiently deliver RNAs in vivo and allow stable gene silencing. For example, a vector-mediated RNAi -inducing gene cassette determined stable gene silencing thanks to the stable insertion of the vector into the host genome [120].

In addition, gene silencing can be tumor-targeted by modifying the capsid structures that regulate viral cell tropism thus driving viral vectors in specific cell types [121]. In the past years, viral vector-miRNA delivery has been used for the treatment of many diseases including cancer. For example, in a recent study an adeno-associated viral vector was used to restore the expression of $m i R-122 a$ in a murine model of hepatocellular carcinoma using a virus with a strong liver tropism [122].

However, viral vectors have different limitations and adverse effects that should be solved before they can be used routinely in humans. In fact, high transgene expression can affect cell viability and sncRNA overexpression can result in cellular toxicity, off-target effects as well as in the induction of the immune interferon pathway. In addition, the recurrence of non-specific gene silencing 


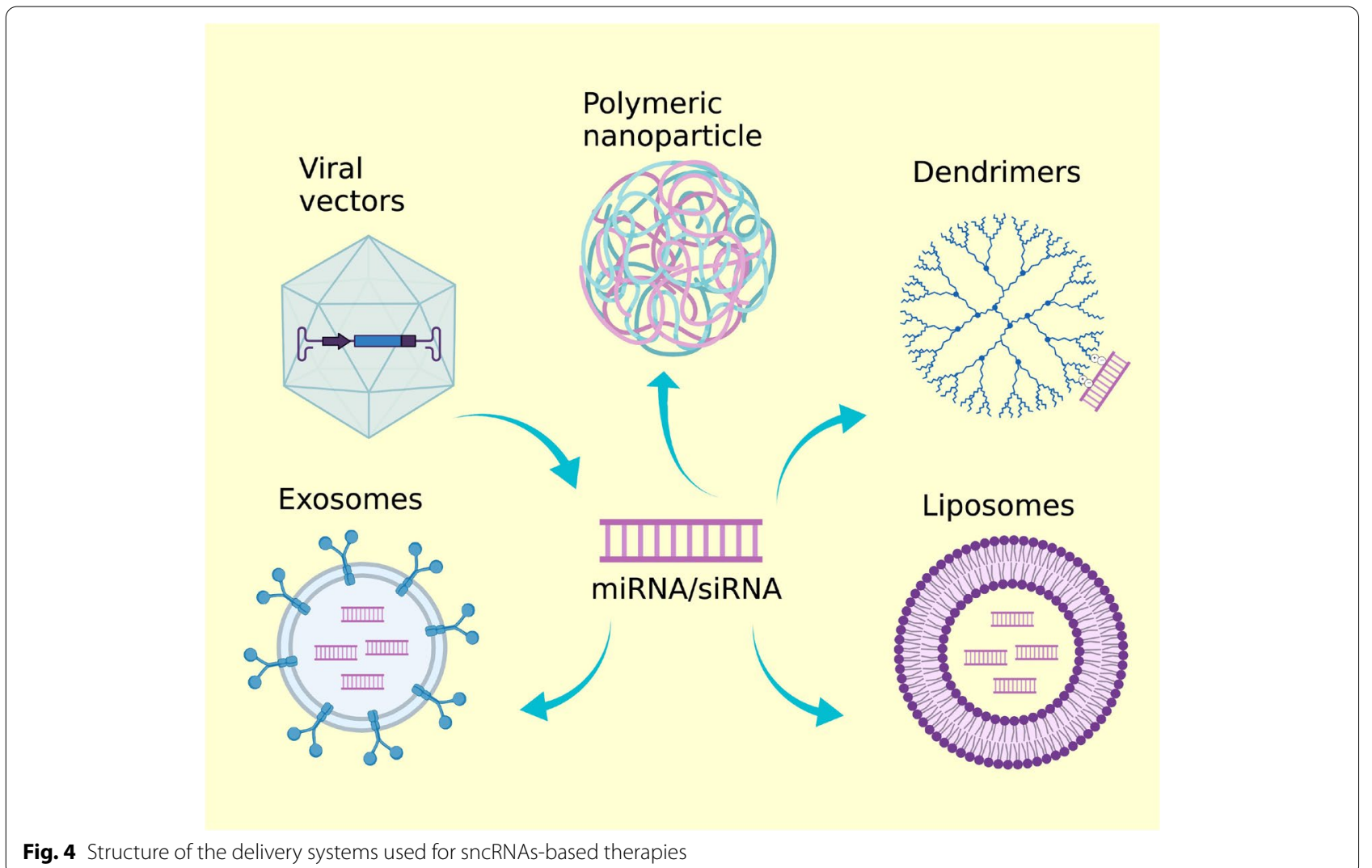

and off-targeting issues makes it difficult to use them in clinical applications. Finally, to be used in therapy, viruses must be genetically engineered to remove their virulence and to alter their natural tropism toward specific cell types by modifying proteins of the viral capsid.

Despite the modifications that can be done on viral vectors, other serious safety concerns such as the risk of insertional mutagenesis in case of lentiviruses [123] and high immunogenicity in case of adenoviruses [124] stimulated scientists to develop non-viral vectors for siRNA and miRNA delivery with a better safety profile.

Among non-viral systems, polymer-based and lipidbased systems represent different and very flexible tools for RNA delivery. These systems can be modified in different ways to improve serum stability, half-life, and they can be used for site-specific delivery by using targeting ligands during formulation preparation. In addition, polymers and lipids have the advantage of being positively charged and thus they can associate to the negatively charged RNA [122, 125].

Among polymers, the cationic PEI (polyethylenimine) has been widely investigated for many years for the high efficiency delivery in vivo [126]. PEI acts as coating providing a net cationic charge while forming polyplexes with miRNA and siRNA, thus protecting them from degradation mediated by serum enzymes. It also enhances the introduction in the cell through the interaction with anionic cell membrane polysaccharides [127, 128]. Nanosized polyplexes are easy to prepare and endocytosis can facilitate their cellular uptake. The limitation of the use of this delivery system is represented by the possible interaction of the coating with serum proteins. In addition, due to its high toxicity PEI has been started to be used in clinics only after the development of specific modifications [126].

Polyphosphazenes are other synthetic polymers showing high biocompatibility and chemical flexibility that can be modified to achieve targeted release to the site of action. Different studies report their use to deliver sncRNAs in therapeutics [129].

Other natural cationic polymers such as PLGA (Poly (lactic-co-glycolic acid)) and cyclodextrins are considered as safe sncRNAs delivery systems.

PLGA is a synthetic biodegradable polymer-FDA approved, that has lower or absent toxicity. The advantage of PLGA is that with this polymer it is possible to modulate the drug release by changing its molecular weight and composition.

Cyclodextrins, cyclic oligomers of glucose, are other polymers widely accepted for human pharmaceutical 
formulations due to low toxicity, high stability and lack of immune stimulation.

Both PLGA and cyclodextrins have the advantage that they can be used to form nanoparticles in which sncRNAs can be loaded [130,131].

Other attractive polymers used are the dendrimers, multi-branched polymers that contain a core surrounded by repeated iterations - the branches. Branches can have functional groups which can be modified for ligand attachment. Their structure allows them to complex many small RNAs molecules. Dendrimers can overcome intra- and extracellular barriers and have been used in different therapeutic fields [132, 133].

Unlike polymers, the use of lipids in vivo does not represent a good choice system due to toxicity, nonspecific uptake, and to the burst of inflammatory and immune responses. Often lipids nanoparticles are liposomes combined to PEG. Other strategies are based on the use of liposomes and protamine containing small RNA complexed to hyaluronic acid [134].

An evolution of cationic lipids used for sncRNAs delivery is represented by SNALPs (Stable nucleic acid lipid particles). These nanoparticles are characterized by the presence of an ionizable amino lipid into the lipid vesicles that facilitate the encapsulation of sncRNA with increase of serum stability. SNALPs have been used for the delivery of siRNA and miRNAs in different tumors and some SNALP-based formulations currently in clinical trials [135].

More recently, a combined system made of polymers and lipids has been developed and used for in vivo delivery of small RNAs. This system - named lipolyplexes - combines the advantageous characteristics of both molecules [136]. For example, Patisiran is a lipolyplexes clinically approved encapsulating siRNA directed against transthyretin mRNA. This delivery system after entering into the cell allows siRNA diffusion in the cytosol thanks to interactions between cationic lipids of the nanoparticles and anionic lipids of the endosomal membrane [137].

Lipoplexes are the most used delivery vehicles for sncRNA molecules and represent a promising delivery system since it is possible to modulate transfection efficiency and toxicity by changing the structure composition and /or the properties of the final formulation [138-140].

The commercial lipid-based systems for in vitro transfection are frequently used to deliver noncoding RNAs in cells with high efficiency, and in vivo performance has been recently improved. Thanks to the structural optimization developed in the last years, this delivery system has become highly efficient in capacity and in targeting specific cells. Interestingly, some lipoplexes formulations are currently investigated in clinical trials (ClinicalTrials. gov) for cancer treatment (Atu027, EphA2, DCR-MYC, TKM-08030).

A recent growing interest is related to the use of exosomes as sncRNAs delivery system. Exosomes can be viewed as the most challenging small RNA delivery systems since they can be efficient in drug delivery and can be produced at large-scale. Exosomes can be considered as lipid-based nanocarriers [141] being small membrane vesicles that are generated from cells. Discovered thirty years ago as components of the cellular waste disposal mechanism, exosomes are known to play a key role in the cellular signaling of cancer cells. Their formation lead to the production of vehicles charged with molecules coming from their cell of origin $[142,143]$.

Exosomes, being natural carriers of nucleic acids and proteins, represent the best candidate to deliver miRNAs and siRNAs for therapeutic purposes. In fact, due to their biological origin they are biocompatible and lack toxicity. Moreover, their small size allows them to easily encapsulate small RNA molecules through traditional transfection methods. In addition, due to their cellular nature exosomes escape phagocytic degradation and are naturally stable. Moreover, exosomes can cross the bloodbrain barrier, thus can be useful also to develop brain cancer therapeutics [144].

The advantage in using exosomes as delivery systems is that they can be used for large-scale production with highly reproducible results. Finally, with exosomes it is possible to improve target specificity through genetic modification of their surface ligands.

Recent studies reported the efficacy of exosomemediated delivery of siRNAs in different cancers such as breast cancer [145], leukemia [146] and glioma [147].

Finally, the relationship between exosomal miRNA deregulation and cancer can be used to develop targeted exosome-based miRNAs delivery. In fact, cancer exosomes have the ability to convert specific pre-miRNAs to mature-miRNAs, thus enriching them. The identification of miRNA exosome profile for a specific cancer allows the design of specific therapeutic exosomes to deliver the specific sncRNAs.

The development of delivery systems based on exosomes represents a very promising strategy to use these molecules in cancer therapeutics.

\section{Conclusions}

Cancer is often a complex and multifactorial disease that nowadays still presents a lot of limitations in its treatment. Most of the therapies actually used to treat cancer are effective but present many side effects and their function can easily be reduced by multi-drug resistance. RNAi therapeutics are a very interesting and challenging possibility to treat cancer acting on the primary 
cause of its genesis, due to their possibility to target the mRNA expression of genes that are primarily involved in carcinogenesis. A lot of pre-clinical studies have demonstrated that miRNA and siRNA could be useful in diagnosing and treating cancer, but unfortunately most of the clinical trials involving them are still in phase I or II. A series of limitations have to be overcome to let these therapeutics be fully functional to treat this disease that still affects a lot of people worldwide. Despite all the limitations that slow down the approval of sncRNA-based therapeutics for cancer treatment, many studies have highlighted their functional use in this field. Specifically, miRNAs have been shown to be specific and sensible diagnostic and prognostic markers, easy to be dosed due to their presence in body fluids, while siRNAs are more specific molecules that can potentially inhibit the expression of any gene involved in cancer, without the limitations that characterize miRNAs. Since their discovery, miRNAs and siRNAs have become an important object of interest, and are still fascinating researchers from all medical fields. To date two sncRNAs-based therapeutics have been approved in clinics: ONPATTRO ${ }^{\circledR}$ (Patisiran) for treatment of polyneuropathy caused by hereditary transthyretin-mediated amyloidosis [148] and GIVLAARITM $^{\circledR}$ (Givosiran) for the treatment of acute hepatic porphyria [149]. The increasing number of clinical trials with sncRNAs in cancer will allow to develop novel and efficient therapeutics approaches.

In clinical oncology the identification of potential prognostic factors -patient-related characteristics -is essential to assess a valuable prognosis, often independent from the treatment. A meta-analysis can be useful to identify predictive factors, thus allowing clinicians to select patient subgroups that possibly will benefit more from a specific treatment. To this aim the application of systematic meta-analysis will be important to evaluate the quality of a trial, and to quantify the overall treatment efficacy.

The importance of meta-analysis is that results are applicable to a broad spectrum of topics, including biomarkers, genetic factors, diagnosis, and treatment.

Meta-analysis application in clinical trials of sncRNAs therapeutics can be extended to other important areas, such as prognostic models, which are very important in oncology and should include individual studies. In addition, prognostic factors can be evaluated combining meta-analysis data and the data availablein realworld database [150]. The integration of these data might improve the construction of prognostic models and used to assess the efficacy and chose the better sncRNAs -based anticancer therapy.

Noncoding RNA therapeutics by modulating gene expression can act on several targets usually not reached by traditional chemical drugs that block the activity of specific proteins or enzymes. For this reason, they are a promising possibility to overcome the inability and the side effects related to the use of chemotherapeutics.

\begin{abstract}
Abbreviations
AGO: Argonaute; Antagomir: antisense oligonucleotides; Anti-oncomirs: miRNA tumor-suppressors; AR: Androgen receptor; ASOs: antisense oligonucleotides; $\mathrm{BH} 2$ : BCl-2 homology domain 2; CLL: chronic lymphocytic leukemia; DGCR8: DiGeorge syndrome critical region 8; DOPC: 1,2-dioleoyl-sn-glycero3-phosphatidylcholine; DsiRNA: Dicer-substrate small interfering RNA; dsRNA: double-stranded RNA; DUF283: Domain of unknown function; GW-bodies: Glycine-Tryptophan bodies; KSP: kinesin spindle protein; LNAs: Locked nucleic acids; IncRNAs: long non-coding RNAs; Mimic: miRNA re-introduced in the cell; miRNAs: microRNA; PNA: Peptide nucleic acids; PD-1: Programmed death-protein 1; PD-L1/2: Programmed death-protein ligands 1 and 2; PEI: polyethylenimine; piRNAs: Piwi-interacting RNAs; PIWI: P-element Induced Wimpy; PKN3: Protein kinase N3; PLGA: Poly lactic-co-glycolic acid; PLKs: Pololike kinases; RISC: RNA-induced silencing complex; RNAi: RNA interference; RNR: Ribonucleotide reductase; RRM2: Ribonucleoside reductase M2; SNALPS: Stable nucleic acid lipid particles; SNAs: spherical nucleic acids; UTR: untranslated region;VEGF-A: vascular endothelial growth factor-A.
\end{abstract}

\section{Supplementary Information}

The online version contains supplementary material available at https://doi. org/10.1186/s13046-021-02193-1.

Additional file 1: Supplemental Figure 1. Flow diagram summarizing how the study was conducted. A) Literature search was performed using PubMed and Web of Science databases using as keywords: small non coding RNAs, microRNAs, small interfering RNAs and cancer. Research reports, review articles and articles published up to October 2021 were evaluated. B) Clinical trials search was performed using the "Clinicaltrials.gov" database insertingthe same keywords as in A). Only the studies using miRNAs or siRNAs as drug were selected. Clinical trials in which miRNAs or siRNAs were evaluated as diagnostic or predictive tests were excluded.

Additional file 2: Supplemental Table 1. sncRNAs in prostate cancer preclinical trials.

Acknowledgements

RC's PhD fellowship in Biology is supported by CNR/IRCCS Neuromed/Università degli Studi di Napoli Federico II "Dottorati Industriali" (XXXVI Cycle). We thank Chiara Nobile and Valentina Brasiello for their editing assistance. We thank Maria Rosaria Aletta for her bibliographic assistance and Maria Rosaria Chiummo for her help in reviewing the manuscript.

Figures were created using BioRender.com

\section{Authors' contributions}

$\mathrm{RC}$ and SF equally contributed to this work. RC and SF writing the manuscript SC conceived the manuscript and supervised it. All authors read and approved the final manuscript.

Funding

This work was supported by CNR project NUTR-AGE (DSB.AD004.271).

Availability of data and materials

Not applicable.

\section{Declarations}

Ethics approval and consent to participate

Not applicable. 


\section{Consent for publication}

All authors consent to publication.

\section{Competing interests}

The authors declare that they have no competing interests.

\section{Author details}

'Institute of Biosciences and BioResources-UOS Naples CNR, via P. Castellino, 111-80131 Naples, Italy. ${ }^{2}$ IRCCS Neuromed, Pozzilli, IS, Italy.

Received: 14 October 2021 Accepted: 23 November 2021

Published online: 04 December 2021

\section{References}

1. Grillone K, Riillo C, Scionti F, Rocca R, Tradigo G, Guzzi PH, et al. Noncoding RNAs in cancer: platforms and strategies for investigating the genomic "dark matter". J Exp Clin Cancer Res. 2020;39(117).

2. Costa FF. Non-coding RNAs: new players in eukaryotic biology. Gene. 2005;357:83-94

3. Toden S, Zumwalt TJ, Goel A. Non-coding RNAs and potential therapeutic targeting in cancer. Biochim Biophys Acta Rev Cancer. 2021:1875:188491.

4. Pauli A, Rinn JL, Schier AF. Non-coding RNAs as regulators of embryogenesis. Nat Rev Genet. 2011;12:136-49.

5. Esteller M. Non-coding RNAs in human disease. Nat Rev Genet. 2011;12:861-74.

6. Carthew RW, Sontheimer EJ. Origins and mechanisms of miRNAs and siRNAs. Cell. 2009;136:642-55.

7. Lee RC, Feinbaum RL, Ambros V. The C. elegans heterochronic gene lin-4 encodes small RNAs with antisense complementarity to lin-14. Cell. 1993;75:843-54.

8. Bartel DP. MicroRNAs: genomics, biogenesis, mechanism, and function Cell. 2004;116:281-97.

9. Novina CD, Sharp PA. The RNAi revolution. Nature. 2004;430:161-4

10. Rupaimoole R, Slack FJ. MicroRNA therapeutics: towards a new era for the management of cancer and other diseases. Nat Rev Drug Discov. 2017;16:203-22.

11. Senapati D, Patra BC, Kar A, Chini DS, Ghosh S, Patra S, et al. Promising approaches of small interfering RNAs (siRNAs) mediated cancer gene therapy. Gene. 2019;719:144071.

12. Davis S, Lollo B, Freier S, Esau C. Improved targeting of miRNA with antisense oligonucleotides. Nucleic Acids Res. 2006;34:2294-304.

13. Li SM, Wu HL, Yu X, Tang K, Wang SG, Ye ZQ, et al. The putative tumour suppressor miR-1-3p modulates prostate cancer cell aggressiveness by repressing E2F5 and PFTK1. J Exp Clin Cancer Res. 2018;37(219).

14. Xu Y, Chen W, Liang J, Zeng X, Ji K, Zhou J, et al. The miR-1185-2-3pGOLPH3L pathway promotes glucose metabolism in breast cancer by stabilizing p53-induced SERPINE1. J Exp Clin Cancer Res. 2021:40(47).

15. Iorns E, Lord CJ, Turner N, Ashworth A. Utilizing RNA interference to enhance cancer drug discovery. Nat Rev Drug Discov. 2007;6:556-68.

16. Mello CC, Conte D. Revealing the world of RNA interference. Nature. 2004:431:338-42.

17. Meister G, Landthaler M, Patkaniowska A, Dorsett Y, Teng G, TuschI T. Human Argonaute2 mediates RNA cleavage targeted by miRNAs and siRNAs. Mol Cell. 2004:15:185-97.

18. Tomari Y, Zamore PD. Perspective: machines for RNAi. Genes Dev. 2005:19:517-29.

19. Stark A, Bushati N, Jan CH, Kheradpour P, Hodges E, Brennecke J, et al. A single Hox locus in Drosophila produces functional microRNAs from opposite DNA strands. Genes Dev. 2008;22:8-13.

20. Tyler DM, Okamura K, Chung WJ, Hagen JW, Berezikov E, Hannon GJ, et al. Functionally distinct regulatory RNAs generated by bidirectional transcription and processing of microRNA loci. Genes Dev. 2008:22:26-36.

21. Lewis BP, Burge CB, Bartel DP. Conserved seed pairing, often flanked by adenosines, indicates that thousands of human genes are microRNA targets. Cell. 2005;120:15-20.
22. Rodriguez A, Griffiths-Jones S, Ashurst JL, Bradley A. Identification of mammalian microRNA host genes and transcription units. Genome Res. 2004;14:1902-10.

23. Lee Y, Kim M, Han J, Yeom KH, Lee S, Baek SH, et al. MicroRNA genes are transcribed by RNA polymerase II. EMBO J. 2004;23:4051-60.

24. Han J, Lee Y, Yeom KH, Kim YK, Jin H, Kim VN. The Drosha-DGCR8 com plex in primary microRNA processing. Genes Dev. 2004;18:3016-27.

25. Denli AM, Tops BB, Plasterk RH, Ketting RF, Hannon GJ. Processing of primary microRNAs by the microprocessor complex. Nature. 2004:432:231-5.

26. Yi R, Qin Y, Macara IG, Cullen BR. Exportin-5 mediates the nuclear export of pre-microRNAs and short hairpin RNAs. Genes Dev. 2003;17:3011-6.

27. Moore MS, Blobel G. The GTP-binding protein ran/TC4 is required for protein import into the nucleus. Nature. 1993:365:661-3.

28. Elkayam E, Faehnle CR, Morales M, Sun J, Li H, Joshua-Tor L. Multivalent recruitment of human Argonaute by GW182. Mol Cell. 2017;67:646658.e643

29. Behm-Ansmant I, Rehwinkel J Doerks T, Stark A, Bork P, Izaurralde E. mRNA degradation by miRNAs and GW182 requires both CCR4:NOT deadenylase and DCP1:DCP2 decapping complexes. Genes Dev. 2006;20:1885-98.

30. Schwarz DS, Hutvágner G, Du T, Xu Z, Aronin N, Zamore PD. Asymmetry in the assembly of the RNAi enzyme complex. Cell. 2003;115:199-208.

31. Azuma-Mukai A, Oguri H, Mituyama T, Qian ZR, Asai K, Siomi H, et al. Characterization of endogenous human Argonautes and their miRNA partners in RNA silencing. Proc Natl Acad Sci U S A. 2008;105:7964-9.

32. Eulalio A, Behm-Ansmant I, Izaurralde E. P bodies: at the crossroads of post-transcriptional pathways. Nat Rev Mol Cell Biol. 2007;8:9-22.

33. Hamilton AJ, Baulcombe DC. A species of small antisense RNA in posttranscriptional gene silencing in plants. Science. 1999;286:950-2.

34. Vazquez F, Vaucheret H, Rajagopalan R, Lepers C, Gasciolli V, Mallory AC, et al. Endogenous trans-acting siRNAs regulate the accumulation of Arabidopsis mRNAs. Mol Cell. 2004;16:69-79.

35. Golden DE, Gerbasi VR, Sontheimer EJ. An inside job for siRNAs. Mol Cell. 2008;31:309-12.

36. Baulcombe D. RNA silencing in plants. Nature. 2004;431:356-63.

37. Agrawal N, Dasaradhi PV, Mohmmed A, Malhotra P, Bhatnagar RK, Mukherjee SK. RNA interference: biology, mechanism, and applications. Microbiol Mol Biol Rev. 2003;67:657-85.

38. Valencia-Sanchez MA, Liu J, Hannon GJ, Parker R. Control of translation and mRNA degradation by miRNAs and siRNAs. Genes Dev. 2006;20:515-24.

39. Lingel A, Sattler M. Novel modes of protein-RNA recognition in the RNAi pathway. Curr Opin Struct Biol. 2005:15:107-15.

40. Ipsaro JJ, Joshua-Tor L. From guide to target: molecular insights into eukaryotic RNA-interference machinery. Nat Struct Mol Biol. 2015:22:20-8

41. O'Brien J, Hayder H, Zayed Y, Peng C. Overview of MicroRNA biogenesis, mechanisms of actions, and circulation. Front Endocrinol (Lausanne). 2018;9:402

42. Lytle JR, Yario TA, Steitz JA. Target mRNAs are repressed as efficiently by microRNA-binding sites in the $5^{\prime}$ UTR as in the $3^{\prime}$ UTR. Proc Natl Acad Sci U S A. 2007:104:9667-72.

43. Tay Y, Zhang J, Thomson AM, Lim B, Rigoutsos I. MicroRNAs to Nanog, Oct4 and Sox2 coding regions modulate embryonic stem cell differentiation. Nature. 2008;455:1124-8.

44. Zhang Y, Fan M, Zhang X, Huang F, Wu K, Zhang J, et al. Cellular microRNAs up-regulate transcription via interaction with promoter TATA-box motifs. RNA. 2014;20:1878-89.

45. Huntzinger $\mathrm{E}$, Izaurralde $\mathrm{E}$. Gene silencing by microRNAs: contributions of translational repression and mRNA decay. Nat Rev Genet. 2011:12:99-110.

46. Raemdonck K, Vandenbroucke RE, Demeester J, Sanders NN, De Smedt SC. Maintaining the silence: reflections on long-term RNAi. Drug Discov Today. 2008;13:917-31.

47. Brill JV. Screening for cancer: the economic, medical, and psychosocial issues. Am J Manag Care. 2020;26:S300-6.

48. Qiao J, Liu Z, Fu YX. Adapting conventional cancer treatment for immunotherapy. J Mol Med (Berl). 2016;94:489-95.

49. Qian CN, Mei Y, Zhang J. Cancer metastasis: issues and challenges. Chin J Cancer. 2017:36:38. 
50. Sung H, Ferlay J, Siegel RL, Laversanne M, Soerjomataram I, Jemal A, et al. Global cancer statistics 2020: GLOBOCAN estimates of incidence and mortality worldwide for 36 cancers in 185 countries. CA Cancer J Clin. 2021.

51. Ha M, Kim VN. Regulation of microRNA biogenesis. Nat Rev Mol Cell Biol. 2014;15:509-24.

52. Bader AG, Brown D, Stoudemire J, Lammers P. Developing therapeutic microRNAs for cancer. Gene Ther. 2011;18:1121-6.

53. van Rooij $E$, Kauppinen S. Development of microRNA therapeutics is coming of age. EMBO Mol Med. 2014;6:851-64.

54. Layzer JM, McCaffrey AP, Tanner AK, Huang Z, Kay MA, Sullenger BA In vivo activity of nuclease-resistant siRNAs. RNA. 2004;10:766-71.

55. Krützfeldt J, Rajewsky N, Braich R, Rajeev KG, Tuschl T, Manoharan $M$, et al. Silencing of microRNAs in vivo with 'antagomirs'. Nature. 2005;438:685-9.

56. Czech MP. MicroRNAs as therapeutic targets. N Engl J Med. 2006;354:1194-5.

57. Lennox KA, Behlke MA. Chemical modification and design of antimiRNA oligonucleotides. Gene Ther. 2011;18:1111-20.

58. Obad S, dos Santos CO, Petri A, Heidenblad M, Broom O, Ruse C, et al. Silencing of microRNA families by seed-targeting tiny LNAs. Nat Genet. 2011:43:371-8.

59. Yoshioka K, Kunieda T, Asami Y, Guo H, Miyata H, Yoshida-Tanaka K, et al. Highly efficient silencing of microRNA by heteroduplex oligonucleotides. Nucleic Acids Res. 2019;47:7321-32.

60. Fabani MM, Abreu-Goodger C, Williams D, Lyons PA, Torres AG, Smith KG, et al. Efficient inhibition of miR-155 function in vivo by peptide nucleic acids. Nucleic Acids Res. 2010;38:4466-75.

61. Ebert MS, Neilson JR, Sharp PA. MicroRNA sponges: competitive inhibitors of small RNAs in mammalian cells. Nat Methods. 2007:4:721-6.

62. Lee JB, Hong J, Bonner DK, Poon Z, Hammond PT. Self-assembled RNA interference microsponges for efficient siRNA delivery. Nat Mater. 2012;11:316-22

63. Wang Z. The principles of MiRNA-masking antisense oligonucleotides technology. Methods Mol Biol. 2011;676:43-9.

64. Walton SP, Wu M, Gredell JA, Chan C. Designing highly active siRNAs for therapeutic applications. FEBS J. 2010;277:4806-13.

65. Gantier MP, Williams BR. The response of mammalian cells to doublestranded RNA. Cytokine Growth Factor Rev. 2007;18:363-71.

66. Chaudhary A, Srivastava S, Garg S. Development of a software tool and criteria evaluation for efficient design of small interfering RNA. Biochem Biophys Res Commun. 2011:404:313-20.

67. He F, Han Y, Gong J, Song J, Wang H, Li Y. Predicting siRNA efficacy based on multiple selective siRNA representations and their combination at score level. Sci Rep. 2017;7:44836.

68. Meister $\mathrm{G}$. Argonaute proteins: functional insights and emerging roles. Nat Rev Genet. 2013;14:447-59.

69. Petri S, Meister G. siRNA design principles and off-target effects. Methods Mol Biol. 2013;986:59-71.

70. Wang X, Varma RK, Beauchamp L, Magdaleno S, Sendera TJ. Selection of hyperfunctional siRNAs with improved potency and specificity. Nucleic Acids Res. 2009;37:e152.

71. Birmingham A, Anderson E, Sullivan K, Reynolds A, Boese Q, Leake $D$, et al. A protocol for designing siRNAs with high functionality and specificity. Nat Protoc. 2007:2:2068-78.

72. Jackson AL, Burchard J, Schelter J, Chau BN, Cleary M, Lim L, et al. Widespread siRNA "off-target" transcript silencing mediated by seed region sequence complementarity. RNA. 2006;12:1179-87.

73. Saxena S, Jónsson ZO, Dutta A. Small RNAs with imperfect match to endogenous mRNA repress translation. Implications for off-target activity of small inhibitory RNA in mammalian cells. J Biol Chem. 2003:278:44312-9.

74. Grimm D, Streetz KL, Jopling CL, Storm TA, Pandey K, Davis CR, et al. Fatality in mice due to oversaturation of cellular microRNA/short hairpin RNA pathways. Nature. 2006;441:537-41.

75. Lin X, Ruan X, Anderson MG, McDowell JA, Kroeger PE, Fesik SW, et al. siRNA-mediated off-target gene silencing triggered by a $7 \mathrm{nt}$ complementation. Nucleic Acids Res. 2005;33:4527-35

76. Jackson AL, Linsley PS. Recognizing and avoiding siRNA off-target effects for target identification and therapeutic application. Nat Rev Drug Discov. 2010;9:57-67.
77. Doench JG, Petersen CP, Sharp PA. siRNAs can function as miRNAs. Genes Dev. 2003;17:438-42.

78. Vannini I, Fanini F, Fabbri M. Emerging roles of microRNAs in cancer. Curr Opin Genet Dev. 2018;48:128-33.

79. Zhang S, Cheng Z, Wang Y, Han T. The risks of miRNA therapeutics: in a drug target perspective. Drug Des Devel Ther. 2021;15:721-33.

80. Cimmino A, Calin GA, Fabbri M, Iorio MV, Ferracin M, Shimizu M, et al. miR-15 and miR-16 induce apoptosis by targeting BCL2. Proc Natl Acad Sci U S A. 2005:102:13944-9.

81. Lovat F, Fassan M, Gasparini P, Rizzotto L, Cascione L, Pizzi M, et al. miR-15b/16-2 deletion promotes B-cell malignancies. Proc Natl Acad Sci U S A. 2015;112:11636-41.

82. Reid G, Kao SC, Pavlakis N, Brahmbhatt H, MacDiarmid J, Clarke S, et al. Clinical development of TargomiRs, a miRNA mimic-based treatment for patients with recurrent thoracic cancer. Epigenomics. 2016:8:1079-85.

83. Tili E, Michaille JJ, Wernicke D, Alder H, Costinean S, Volinia S, et al. Mutator activity induced by microRNA-155 (miR-155) links inflammation and cancer. Proc Natl Acad Sci U S A. 2011;108:4908-13.

84. Kopp KL, Ralfkiaer U, Gjerdrum LM, Helvad R, Pedersen IH, Litman T, et al. STAT5-mediated expression of oncogenic miR-155 in cutaneous T-cell lymphoma. Cell Cycle. 2013;12:1939-47.

85. Querfeld C, Pacheco T, Foss F, Halwani A, Porcu P, Seto A, et al. Preliminary results of a phase 1 trial evaluating MRG-106, a synthetic microRNA antagonist (LNA antimiR) of microRNA-155, in patients with CTCL. Blood. 2016;128.

86. Wang J, Dan G, Zhao J, Ding Y, Ye F, Sun H, et al. The predictive effect of overexpressed miR-34a on good survival of cancer patients: a systematic review and meta-analysis. Onco Targets Ther. 2015;8:2709-19.

87. Daige $\mathrm{CL}$, Wiggins JF, Priddy L, Nelligan-Davis T, Zhao J, Brown D. Systemic delivery of a miR34a mimic as a potential therapeutic for liver cancer. Mol Cancer Ther. 2014;13:2352-60.

88. Hong DS, Kang YK, Borad M, Sachdev J, Ejadi S, Lim HY, et al. Phase 1 study of MRX34, a liposomal miR-34a mimic, in patients with advanced solid tumours. Br J Cancer. 2020;122:1630-7.

89. Aleku M, Schulz P, Keil O, Santel A, Schaeper U, Dieckhoff B, et al. Atu027, a liposomal small interfering RNA formulation targeting protein kinase N3, inhibits cancer progression. Cancer Res. 2008:68:9788-98

90. Schultheis B, Strumberg D, Santel A, Vank C, Gebhardt F, Keil O, et al. First-in-human phase I study of the liposomal RNA interference therapeutic Atu027 in patients with advanced solid tumors. J Clin Oncol. 2014;32:4141-8.

91. Schultheis B, Strumberg D, Kuhlmann J, Wolf M, Link K, Seufferlein T, et al. A phase Ib/lla study of combination therapy with gemcitabine and Atu027 in patients with locally advanced or metastatic pancreatic adenocarcinoma. J Clin Oncol. 2016;34.

92. Fleming JB, Shen GL, Holloway SE, Davis M, Brekken RA. Molecular consequences of silencing mutant K-ras in pancreatic cancer cells: justification for K-ras-directed therapy. Mol Cancer Res. 2005;3:413-23.

93. Réjiba S, Wack S, Aprahamian M, Hajri A. K-ras oncogene silencing strategy reduces tumor growth and enhances gemcitabine chemotherapy efficacy for pancreatic cancer treatment. Cancer Sci. 2007;98:1128-36.

94. Zorde Khvalevsky E, Gabai R, Rachmut IH, Horwitz E, Brunschwig Z, Orbach A, et al. Mutant KRAS is a druggable target for pancreatic cancer. Proc Natl Acad Sci U S A. 2013;110:20723-8.

95. Golan T, Hubert A, Shemi A, Segal A, Dancour A, Khvalevsky E, et al. A phase I trial of a local delivery of siRNA against k-ras in combination with chemotherapy for locally advanced pancreatic adenocarcinoma. J Clin Oncol. 2013;31

96. Varghese A, Ang C, Dimaio C, Javle M, Gutierrez M, Yarom N, et al. A phase II study of siG12D-LODER in combination with chemotherapy in patients with locally advanced pancreatic cancer (PROTACT). J Clin Oncol. 2020;38

97. Lee MS, Pant S. Personalizing medicine with germline and somatic sequencing in advanced pancreatic Cancer: current treatments and novel opportunities. Am Soc Clin Oncol Educ Book. 2021;41:1-13.

98. Sun W, Su Q, Cao X, Shang B, Chen A, Yin H, et al. High expression of polo-like kinase 1 is associated with early development of hepatocellular carcinoma. Int J Genomics. 2014:2014:312130. 
99. Liao X, Tang S, Thrasher JB, Griebling TL, Li B. Small-interfering RNAinduced androgen receptor silencing leads to apoptotic cell death in prostate cancer. Mol Cancer Ther. 2005:4:505-15.

100. Lee JB, Zhang K, Tam YY, Tam YK, Belliveau NM, Sung VY, et al. Lipid nanoparticle siRNA systems for silencing the androgen receptor in human prostate cancer in vivo. Int J Cancer. 2012;131:E781-90.

101. Noblitt LW, Bangari DS, Shukla S, Knapp DW, Mohammed S, Kinch MS, et al. Decreased tumorigenic potential of EphA2-overexpressing breast cancer cells following treatment with adenoviral vectors that express EphrinA1. Cancer Gene Ther. 2004;11:757-66.

102. Duxbury MS, Ito H, Zinner MJ, Ashley SW, Whang EE. EphA2: a determinant of malignant cellular behavior and a potential therapeutic target in pancreatic adenocarcinoma. Oncogene. 2004:23:1448-56.

103. Wagner MJ, Mitra R, McArthur MJ, Baze W, Barnhart K, Wu SY, et al. Preclinical mammalian safety studies of EPHARNA (DOPC Nanoliposomal EphA2-targeted siRNA). Mol Cancer Ther. 2017;16:1114-23.

104. Naing A, Lopez-Berestein G, Fu S, Tsimberidou A, Pant S, Piha-Paul S, et al. EphA2 gene targeting using neutral liposomal small interfering RNA (EPHARNA) delivery: a phase I clinical trial. J Clin Oncol. 2017;35.

105. Stegh AH, Kesari S, Mahoney JE, Jenq HT, Forloney KL, Protopopov A, et al. Bcl2L12-mediated inhibition of effector caspase-3 and caspase-7 via distinct mechanisms in glioblastoma. Proc Natl Acad Sci U S A. 2008;105:10703-8.

106. Kumthekar P, Ko CH, Paunesku T, Dixit K, Sonabend AM, Bloch O, et al. A first-in-human phase 0 clinical study of RNA interference-based spherical nucleic acids in patients with recurrent glioblastoma. Sci Transl Med. 2021:13.

107. Tabernero J, Shapiro GI, LoRusso PM, Cervantes A, Schwartz GK, Weiss GJ, et al. First-in-humans trial of an RNA interference therapeutic targeting VEGF and KSP in cancer patients with liver involvement. Cancer Discov. 2013;3:406-17.

108. Zhan $Y$, Jiang $L$, Jin $X$, Ying $S$, Wu Z, Wang $L$, et al. Inhibiting RRM 2 to enhance the anticancer activity of chemotherapy. Biomed Pharmacother. 2021;133:110996.

109. Zuckerman JE, Gritli I, Tolcher A, Heidel JD, Lim D, Morgan R, et al. Correlating animal and human phase la/lb clinical data with CALAA01, a targeted, polymer-based nanoparticle containing siRNA. Proc Natl Acad Sci U S A. 2014:111:11449-54

110. Miller DM, Thomas SD, Islam A, Muench D, Sedoris K. C-Myc and cancer metabolism. Clin Cancer Res. 2012;18:5546-53.

111. Anthony WT, Kyriakos PP, Amita P, Drew Warren R, Dorothy M, Debra LW, et al. Safety and activity of DCR-MYC, a first-in-class Dicer-substrate small interfering RNA (DsiRNA) targeting MYC, in a phase I study in patients with advanced solid tumors. J Clin Oncol. 2015;33:11006

112. Chen YG, Satpathy AT, Chang HY. Gene regulation in the immune system by long noncoding RNAs. Nat Immunol. 2017;18:962-72.

113. Banchereau J, Steinman RM. Dendritic cells and the control of immunity. Nature. 1998;392:245-52.

114. Roeven MW, Hobo W, van der Voort R, Fredrix H, Norde WJ, Teijgeler $\mathrm{K}$, et al. Efficient nontoxic delivery of PD-L1 and PD-L2 siRNA into dendritic cell vaccines using the cationic lipid SAINT-18. J Immunother. 2015:38:145-54

115. Dannull J, Haley NR, Archer G, Nair S, Boczkowski D, Harper M, et al. Melanoma immunotherapy using mature DCs expressing the constitutive proteasome. J Clin Invest. 2013;123:3135-45.

116. Salzberg M, Triozzi P, Kooshki M, Alistar A, Bitting R, Fenstermaker J, et al. Adoptive cellular immunotherapy with APN401, autologous Cbl-b-silenced peripheral blood mononuclear cells, in patients with solid tumors. J Clin Oncol. 2016:34.

117. Triozzi P, Kooshki M, Alistar A, Bitting R, Neal A, Lametschwandtner $\mathrm{G}$, et al. Phase I clinical trial of adoptive cellular immunotherapy with APN401 in patients with solid tumors. J ImmunoTher Cancer. 2015;3.

118. Lima J, Cerqueira L, Fiqueiredo C, Oliveira C, Azevedo N. Anti-miRNA oligonucleotides: A comprehensive guide for design. RNA Biol. 2018;15:338-52.

119. Couto LB, High KA. Viral vector-mediated RNA interference. Curr Opin Pharmacol. 2010;10:534-42.

120. Brummelkamp TR, Bernards R, Agami R. A system for stable expression of short interfering RNAs in mammalian cells. Science. 2002:296:550-3.
121. Hajitou A, Trepel M, Lilley CE, Soghomonyan S, Alauddin MM, Marini FC, et al. A hybrid vector for ligand-directed tumor targeting and molecular imaging. Cell. 2006;125:385-98.

122. Della Peruta M, Badar A, Rosales C, Chokshi S, Kia A, Nathwani D, et al. Preferential targeting of disseminated liver tumors using a recombinant adeno-associated viral vector. Hum Gene Ther. 2015;26:94-103.

123. Baum C, Kustikova O, Modlich U, Li Z, Fehse B. Mutagenesis and oncogenesis by chromosomal insertion of gene transfer vectors. Hum Gene Ther. 2006:17:253-63.

124. Atasheva S, Yao J, Shayakhmetov DM. Innate immunity to adenovirus: lessons from mice. FEBS Lett. 2019:593:3461-83.

125. Freitag F, Wagner E. Optimizing synthetic nucleic acid and protein nanocarriers: the chemical evolution approach. Adv Drug Deliv Rev. 2021:168:30-54

126. Urban-Klein B, Werth S, Abuharbeid S, Czubayko F, Aigner A. RNAi-mediated gene-targeting through systemic application of polyethylenimine (PEI)-complexed siRNA in vivo. Gene Ther. 2005;12:461-6.

127. Baumann $V$, Winkler J. miRNA-based therapies: strategies and delivery platforms for oligonucleotide and non-oligonucleotide agents. Future Med Chem. 2014:6:1967-84.

128. Bolcato-Bellemin AL, Bonnet ME, Creusat G, Erbacher P, Behr JP. Sticky overhangs enhance siRNA-mediated gene silencing. Proc Natl Acad Sci U S A. 2007;104:16050-5.

129. Hsu W, Csaba N, Alexander C, Garcia-Fuentes M. Polyphosphazenes for the delivery of biopharmaceuticals. J Appl Polym Sci. 2020;137.

130. Su Y, Zhang B, Sun R, Liu W, Zhu Q, Zhang X, et al. PLGA-based biodegradable microspheres in drug delivery: recent advances in research and application. Drug Delivery. 2021;28:1397-418.

131. Davis M, Brewster M. Cyclodextrin-based pharmaceutics: past, present and future. Nat Rev Drug Discov. 2004;3:1023-35.

132. Madaan K, Kumar S, Poonia N, Lather V, Pandita D. Dendrimers in drug delivery and targeting: drug-dendrimer interactions and toxicity issues. J Pharm Bioallied Sci. 2014;6:139-50.

133. Singh V, Kesharwani P. Dendrimer as a promising nanocarrier for the delivery of doxorubicin as an anticancer therapeutics. J Biomater Sci Polym Ed. 2021:1-28.

134. Wu SY, McMillan NA. Lipidic systems for in vivo siRNA delivery. AAPS J. 2009:11:639-52.

135. Campani V, Salzano G, Lusa S, De Rosa G. Lipid Nanovectors to Deliver RNA Oligonucleotides in Cancer. Nanomaterials. 2016;6.

136. Thanki K, Zeng X, Justesen S, Tejlmann S, Falkenberg E, Van Driessche $E$, et al. Engineering of small interfering RNA-loaded lipidoid-poly (DL-lactic-co-glycolic acid) hybrid nanoparticles for highly efficient and safe gene silencing: a quality by design-based approach. Eur J Pharm Biopharm. 2017;120:22-33.

137. Wood H. FDA approves patisiran to treat hereditary transthyretin amyloidosis. Nat Rev Neurol. 2018;14:570.

138. Berger M, Lechanteur A, Evrard B, Piel G. Innovative lipoplexes formulations with enhanced siRNA efficacy for cancer treatment: where are we now? Int J Pharm. 2021;605:120851.

139. Hasima N, Ozpolat B. Regulation of autophagy by polyphenolic compounds as a potential therapeutic strategy for cancer. Cell Death Dis. 2014:5:e1509.

140. Ozpolat B, Sood AK, Lopez-Berestein G. Liposomal siRNA nanocarriers for cancer therapy. Adv Drug Deliv Rev. 2014;66:110-6.

141. Barile L, Vassalli G. Exosomes: therapy delivery tools and biomarkers of diseases. Pharmacol Ther. 2017:174:63-78.

142. Challagundla KB, Wise PM, Neviani P, Chava H, Murtadha M, Xu T, et al. Exosome-mediated transfer of microRNAs within the tumor microenvironment and neuroblastoma resistance to chemotherapy. J Natl Cancer Inst. 2015;107.

143. Zhang L, Zhang S, Yao J, Lowery FJ, Zhang Q, Huang WC, et al. Microenvironment-induced PTEN loss by exosomal microRNA primes brain metastasis outgrowth. Nature. 2015:527:100-4.

144. Wiklander O, Brennan M, Lotval J, Breakefield X, Andaloussi EL. Advances in therapeutic applications of extracellular vesicles. Sci Transl Med. 2019;11.

145. Forterre A, Wang J, Delcayre A, Kim K, Green C, Pegram M, et al. Extracellular vesicle-mediated in vitro transcribed mRNA delivery for treatment of HER2(+) breast Cancer xenografts in mice by prodrug CB1954 without general toxicity. Mol Cancer Ther. 2020;19:858-67. 
146. Yang Z, Shi J, Xie J, Wang Y, Sun J, Liu T, et al. Large-scale generation of functional mRNA-encapsulating exosomes via cellular nanoporation (vol 4, pg 69, 020). Nature Biomedical Engineering. 2021;5:944-5.

147. Erkan E, Senfter D, Madlener S, Jungwirth G, Strobel T, Saydam N, et al. Extracellular vesicle-mediated suicide $\mathrm{mRNA}$ /protein delivery inhibits glioblastoma tumor growth in vivo. Cancer Gene Ther. 2017;24:38-44.

148. (FDA) UFaDA: ONPATTRO full prescribing information. FDA, Washington DC. 2018. pp. https://www.accessdata.fda.gov/drugsatfda_docs/nda/ 2018/210922Orig210921s210000TOC.cfm.; 2018:https://www.acces sdata.fda.gov/drugsatfda_docs/nda/2018/210922Orig210921s210 000TOC.cfm.

149. (FDA) UFaDA: GIVLAARI (givosiran) Injection full prescribing information. FDA, Washington DC. 2019. pp. https://www.accessdata.fda. gov/drugsatfda_docs/nda/2019/2121940rig212191s212000TOC.cfm; 2019:https://www.accessdata.fda.gov/drugsatfda_docs/nda/2019/ 212194Orig212191s212000TOC.cfm.

150. van den Boorn HG, Abu-Hanna A, Ter Veer E, van Kleef JJ, Lordick F, Stahl $M$, et al. SOURCE: a registry-based prediction model for overall survival in patients with metastatic Oesophageal or gastric Cancer. Cancers (Basel). 2019;11.

\section{Publisher's Note}

Springer Nature remains neutral with regard to jurisdictional claims in published maps and institutional affiliations.

- fast, convenient online submission

- thorough peer review by experienced researchers in your field

- rapid publication on acceptance

- support for research data, including large and complex data types

- gold Open Access which fosters wider collaboration and increased citations

- maximum visibility for your research: over $100 \mathrm{M}$ website views per year

At BMC, research is always in progress.

Learn more biomedcentral.com/submissions 